\title{
Effects of Carbon Hosts on Electrochemical Properties of Lithium-Sulfur Batteries
}

\author{
Tao $\mathrm{Li}^{1,2}$, Hong Bo ${ }^{1,4, *}$, Huawei $\mathrm{Cao}^{2}$, Yanqing Lai ${ }^{1}$, Yexiang Liu ${ }^{1}$, Zixin Huang ${ }^{3}$ \\ ${ }^{1}$ School of Metallurgy and Environment, Central South University, Changsha, Hunan 410083, China \\ ${ }^{2}$ Shenzhen Perfect Power Technology Co., Ltd, Shenzhen, 518057, China \\ ${ }^{3}$ Engineering Research Center of High Performance Battery Materials and Devices, Research Institute \\ of Central South University in Shenzhen, Shenzhen, 518057, China \\ ${ }^{4}$ School of Materials Science and Engineering, Central South University, Changsha, Hunan 410083, \\ China \\ *E-mail: bop_hong@163.com
}

doi: $10.20964 / 2017.06 .41$

Received: 8 January 2017 / Accepted: 9 April 2017 / Published: 12 May 2017

Three kinds of sulfur cathodes based on different carbon hosts were prepared for lithium-sulfur battery. Effects of carbon hosts on composite structure and cell performance were attentively investigated. The hierarchically porous Ketjen black (ECP) owns higher specific surface area and pore volumes than Super P (SP), thus increasing active material utilization, but incapable of restraining rapid capacity fading. When carbon nanotube (CNT) was used for loading sulfur, sulfur can penetrate adequately into CNT and merge with it completely, which forms strong interaction between them. It helps to trap sulfur or reaction intermediates, and accommodates volume changes of sulfur upon cycling. Besides, the highly conductive CNT favors less polarization and better redox reversibility. These factors together promote electrochemical performance of the cathode. A high reversible capacity of $725 \mathrm{mAh}$ $\mathrm{g}^{-1}$ and capacity retention of $\sim 80 \%$ (based on 2nd discharge) is obtained after 100 cycles for the CNT$\mathrm{S}$ composite. The work guides us how to treat rationally the porous structure when considering suitable carbon material for lithium sulfur batteries.

Keywords: carbon nanotube; porous structure; specific surface area; lithium sulfur battery; electrochemical performance

\section{$\underline{\text { FULL TEXT }}$}

(C) 2017 The Authors. Published by ESG (www.electrochemsci.org). This article is an open access article distributed under the terms and conditions of the Creative Commons Attribution license (http://creativecommons.org/licenses/by/4.0/). 